\title{
Smart collection of measurement from moving objects
}

\author{
Ivan Kholod ${ }^{1}$, Nikolay Plokhoy ${ }^{2}$, Mikhail Kupriyanov ${ }^{3}$, Andrey Shorov $^{4}$ \\ Saint Petersburg Electrotechnical University "LETI", Saint Petersburg, Russian Federation \\ ${ }^{1}$ Corresponding author \\ E-mail: ${ }^{1}$ iiholod@mail.ru, ${ }^{2} e l e f u s @ y a n d e x . r u,{ }^{3}$ mikhail.kupriyanov@gmail.com, ${ }^{4} a$ shxz@mail.ru
}

Received 16 May 2017; accepted 29 May 2017

DOI https://doi.org/10.21595/vp.2017.18635

Check for updates

Abstract. This article describes dynamic management's approach of measurement data streams from moving objects. It allows reducing network traffic and distributing computing all around the measurement acquisition environment. For this purpose, as integration technology of measuring devices, conception of a fog computing is being used. In order to make decisions for switching streams, machine learning methods are being implemented. Experiments proved network traffic's great reduction of transmissible measurements.

Keywords: measurement collection, moving objects, distributed machine learning.

\section{Introduction}

Measuring of a moving object (MO) has a great importance in many fields:

- Management of unmanned vehicle uses measurements of: velocity, pressure, vibration signal and other;

- Space science - measurement is used by manned or unmanned spacecraft for data transmission;

- Rocketry measurement equipment forms an integral part of the rocket range assets used to monitor the position and health of a launch vehicle to determine range safety flight termination criteria (Range purpose is for public safety);

- Motor racing - measurement is a key factor in modern motor racing, allowing race engineers to interpret data collected during a test or race and use it to properly tune the car for optimum performance.

Measurements could be received by different measuring devices (MS): Radar, Lidar, optics, telemetry, navigation and etc. They are placed along the object's motion route and can be joined in measurement points (MP) with local point of collected data acquisition and transmission.

Therewith the following problems of data acquisition and processing are arising:

a) Distance barriers of MP from each other and from data acquisition point;

b) Data, received from different MP, are being duplicated, i.e. their visibility distances are crossed;

c) Usage of data channels with low bandwidth (satellite data channels, wireless channels, radio relay lines, cellular communications channel);

d) Management need of both MSs and MO itself requests an analysis of collected data in real time operation mode.

In this regard, new approaches to measurement acquisition are needed that would ensure an appropriate data delivery from MS to processing measurements center in real-time operation mode at accessible data transmission channels.

\section{Typical approach of measurement collection and processing}

During the measurements, all MS work in measurement mode regardless of the object being in their visibility distance. It is necessary that an object was measured right after its entering in MS visibility distance. Therewith measurement data is being transmitted from MS to measurement acquisition center continuously. According to its processing results MS are tuned in measurement process (for example, radar antennas and video camera objectives pointing, etc.) with respect to current and predictable MO parameters (its location, speed and etc.). 
Thus, all MS generate the streams of measurement data in real-time operation mode that are being transmitted by data channels to the data processing center. Therewith they consume energy and transmit data to the processing center increasing the network traffic.

MO visibility distances for each MP are different but might have significant interceptors. In this regard for all the measurements time duplicated data of different quality $(Q)$ is being received from all MP. Fig. 1 shows the described problem. Collected in such manner data has a high percentage of duplicated measurements and noise.

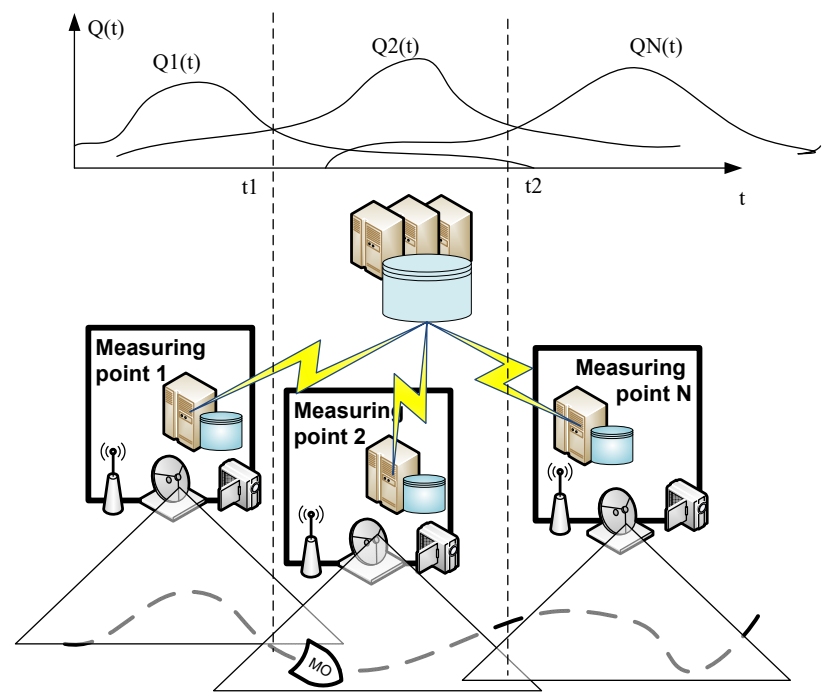

Fig. 1. Typical schema of measurement collection and processing

In measurement processing center, data filters out and combines in single stream of measurements. Based on it the detection of potential danger situations with MO can be performed, and also make decisions for its control.

During the single stream formation in real-time operation mode the following operating procedures are performed at any specific time $t$ :

1) Reception of measurements from MS on each MP;

2) Measurement transmission from MP to processing center;

3) Measurements collection from all MP in processing center;

4) Stream quality assessment received from each MP;

5) Selection of the best measurements and their inclusion into single measurement stream.

Disadvantages of such an approach are the following:

- High network traffic between MP and measurement processing center due to all measurements transmission from MP, even those which in the end will not be included into measurement single stream;

- Great number of calculations in measurement processing center related to quality assessment of each received streams and their connection.

The situation is being worsened by the following factors:

- Single stream must be formed in real-time operation mode to ensure MO operational management;

- It can be several of MO and measurement streams increase depending on their number;

- Data channels with MP might have low bandwidth.

MS connection with data transmission features allows not only collect data but also control them (for example point the camera or radar at the object). Thus, they form Internet of Things $[1,2]$ and Cloud technology [3] is used to build processing center. For this approach, above listed problems appear with number growth of measurement devices and volumes of transmitted data 
the same as with computational and network resources that require continual buildup.

Recently, "fog computing" technology [4] becomes more and more popular. It is, unlike with Cloud computing, with centralized data processing, supposes the part of computing transmission from the central cluster closer to data source. Such approach becomes more popular due to any device intellectualization (its having computing data processor and memory, which allows performing data processing directly on it).

The article proposes to use fog technology and dynamic management of measurement streams for transmission only measurements which are being included into single measurement stream. This allows reducing transmitted data volume and by that to bate demands to transmission environment and data delivery time. Also, it allows distributing computation load between all sites.

\subsection{Proposed approach}

Whole MO measurement system can be represented as a cortege:

$M S=\langle m o, m p c, M P\rangle$,

where: $m o$ - moving object; $m p c$ - a measurement processing center; $M P$ - a great number of measurement points $m p$ :

$M P=\left\{m p_{1}, m p_{2}, \ldots, m p_{r}, \ldots, m p_{m}\right\}$.

Each measurement point $m p_{r}$ contains measurement devices:

$m p_{r}=\left\{m d_{r .1}, m d_{r .2}, \ldots, m d_{r . i}, \ldots, m d_{r . n}\right\}$

Any MO is characterized by some set of parameters:

$m o=\left\{p_{1}, p_{2}, \ldots, p_{k}, \ldots, p_{u}\right\}$

The following can be distinguished among them:

- kinematic parameters: coordinates $(x, y, z)$ and velocity vector $(v x, v y, v z)$;

- state parameters: temperature, vibration, fuel and other parameters transmitted through telemetry;

- image transmitted through the video stream and other.

Parameters transmitted through telemetry and video stream load network traffic the most.

Each measuring device $m d_{r . i}$ at the time $t$ forms a measurement stream $s_{r . i}(t)$ of moving object mo:

$s_{\text {r.i }}(t)=\left\{p_{\text {r.i.1 }}(t), p_{\text {r.i. }}(t), \ldots, p_{\text {r.i.k }}(t), \ldots, p_{\text {r.i.u }}(t)\right\}$,

where $p_{\text {r.i.k }}(t)$ - value of some parameter $p_{k}$ at the time $t$ measured with device $m d_{r . i}$. Each device measures its own set of parameters:

- A radar determines the distance, angle, or velocity of MO;

- A Lidar measures distance to a MO;

- A navigator determinates rectangular or geodesic coordinates and velocity vector of a MO;

- Optic system determinates image and angular coordinates of a MO;

- Sensors measure different parameters which are transmitted by telemetry.

For each measurement stream $s_{r . i}(t)$ we will bring in a stream quality assessment $q_{r . i}(t)$.

Quality assessments for each MS type is performed individually. For example, for radars measurement quality assessment can be determined by returned signal's level. For optical systems quality can be determined by different methods: signal-to-noise ratio, peak signal-to-noise ratio, 
structure similarity and others. Telemetry quality can be determined by built in markers for each fragment, for example, Reed-Solomon codes.

Basic task of measurement system is to form a single stream containing all parameters of $\mathrm{MO}$ measurements at any specific time of the measurements:

$s_{m o}=\left\{s_{m o}\left(t_{1}\right), s_{m o}\left(t_{2}\right), \ldots, s_{m o}\left(t_{j}\right), \ldots, s_{m o}\left(t_{w}\right)\right\}$,

where $s_{m o}\left(t_{j}\right)$ - parameter value set $m o$ at the time $t_{j}$ formed out of all measurements received from measurement devices:

$s_{m o}\left(t_{j}\right)=\operatorname{select}\left(s_{1.1}\left(t_{j}\right), \ldots, s_{r . i}\left(t_{j}\right), \ldots, s_{m . n}\left(t_{j}\right)\right)$

Selection of measurement stream is executed base on stream quality assessment. We propose to select the best stream based on forecasted estimation of quality $q_{r . i}(t)$ for each stream at next time moment. It is possible because assessment for each MS at a time generally occur monotonic. Consequently, projecting quality change of measurement stream, it is possible to determine in advance the moments of time when it is necessary to transmit data for inclusion into the single stream.

The major task is to calculate the $q_{r . i}(t+n)$ for a time period $n$ sufficient for the switching MS. This forecast can be built by different machine learning algorithms, including time series analysis [6]. In this case, the $q_{r . i}(t+n)$ value is approximated to a future period. However, to improve the accuracy of prediction can use additional attributes that affect the quality of the measuring stream. In this case it is possible to form the next vector characterizing the measurement at time station $t$ :

$x q_{r . i}(t+n)=\left\{a_{1}(t), a_{2}(t), \ldots, a_{j}(t), \ldots, a_{m}(t), q(t)\right\}$,

where $a_{j}(t)$ - an attribute, potentially affecting the quality of the measurement. Examples of such attributes may be:

- Distance from MS to the MO;

- The illuminance in the area of MO;

- Moving velocity of MO, etc.

In practical these attributes for $\mathrm{MO}$ are kinematic parameters which are measured together with video and telemetry. In general case, the composition of these attributes may be different and must be determined for each set of MO and MS separately.

The set of all vectors represent an ordered sequence of time from the start of measurement $t=0$ to the end of it $t=w$ :

$X=\left\{x q_{r . i}(0), x q_{r . i}(1), \ldots, x q_{r . i}(t), \ldots, x q_{r . i}(t+n), \ldots, x q_{r . i}(w)\right\}$

The task is finding quality measurement evaluation function (prediction model) for each MS on this vector:

$f: X \rightarrow Q$

Such function can be constructed based on the results of previous measurements of this kind of objects MS data. In this case, for each $x q_{r . i}(t)$ is known for the quality assessment of $q_{r . i}(t)$. Furthermore, the function may be adjusted during the measurements for already performed measurements.

In our approach, a single stream forming $s_{m o}(t)$, at each time $t$ is performed distributed:

(1) Each MD $m d_{r . i}$, placed on the MP $m p_{r}$ performs: 
- Receiving of a measurement stream $s_{r . i}(t)$;

- Transfer of the measurement stream to the MP $m p_{r}$;

(2) Each MP $m p_{r}$ performs:

- Receiving of the measurement stream from each MD $m d_{r . i}$;

- Estimation of the quality $q_{r . i}(t)$ of the measurement stream from each MD;

- Receiving of a command ('send'/'stop') from MPC;

- If received command 'send' then sending of the measurement stream $s_{r . i}(t)$ and quality $q_{r . i}(t)$ for it; else sending of the measurements stream quality $q_{r . i}(t)$ only;

(3) Measurement processing center MPC performs two parallel processes:

- Forecast of quality assessment for each msr. $i$ in which is performed:

- Receiving of the measurements stream qualities $q_{r . i}(t)$ from all MP and measurement stream $s_{m o}(t)$ (for example kinematic parameters) from the best MP $m p_{r}$; model;

- Prediction of quality assessment $x q_{r . i}(t+n)$ for each MD $m d_{r . i}$ using the prediction

- Selection MD $m d_{r . i}$ with the best measurements stream quality $x q_{r . i}(t+n)$;

Sending command 'send' to selected MD $m d_{r . i}$ and commands 'stop' to other MDs;

- Formation of a single measurement stream:

- Receiving of the best measurement stream $s_{r . i}(t)$;

- Including the measurement stream $s_{r . i}(t)$ into a single stream $s_{m o}$.

\section{Experimental results}

As an example, consider the measurement of the parameters of the flying object. According to MO flight path there are three MP each of which includes the following MS:

- Radar measuring kinematic parameters;

- Telemetry.

As the parameters affecting the quality of the measurements are selected:

- For the radar: the distance to the MO, the angle of the object, the velocity of travel of MO;

- For telemetry: distance, angle.

For training was used information from 2 MPs with a measuring duration of 12.5 minutes (3000 measurements each of $0.25 \mathrm{sec}$ ). We used Weka library [6] and the different classifier mining models were trained:

- Regression by discretization [7];

- Decision tree-M5PBase [8].

Experiments were carried out for options:

- The collection of all the information in the processing center;

- Dynamic collection managed by different models.

For each of the variants was measured traffic from 2, 4 and $6 \mathrm{MP}$ at the muster point and compared one stream obtained by collecting all the streams in a collection center.

Table 1. Experimental results

\begin{tabular}{|c|c|c|c|c|}
\hline Approach & $\begin{array}{l}\text { Mining model } \\
\text { correlation }\end{array}$ & $\begin{array}{c}\text { Number of } \\
\text { MPs }\end{array}$ & $\begin{array}{c}\text { Network traffic } \\
(\mathrm{Mb})\end{array}$ & $\begin{array}{c}\text { Quality of single } \\
\text { stream }\end{array}$ \\
\hline \multirow{3}{*}{ Typical approach } & \multirow{3}{*}{ 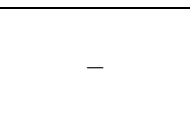 } & 2 & 700 & \multirow{3}{*}{$93 \%$} \\
\hline & & 4 & 1300 & \\
\hline & & 6 & 2100 & \\
\hline \multirow{3}{*}{$\begin{array}{c}\text { Proposed by regression } \\
\text { model }\end{array}$} & \multirow{3}{*}{0.9986} & 2 & 357 & \multirow{3}{*}{$90 \%$} \\
\hline & & 4 & 382 & \\
\hline & & 6 & 400 & \\
\hline \multirow{3}{*}{$\begin{array}{c}\text { Proposed by M5PBase } \\
\text { model }\end{array}$} & \multirow{3}{*}{0.991} & 2 & 351 & \multirow{3}{*}{$89 \%$} \\
\hline & & 4 & 380 & \\
\hline & & 6 & 398 & \\
\hline
\end{tabular}


The experimental results showed a significant reduction of traffic transmitted over the network by using an approach with dynamic control flow. At the same time, the quality of a single flow varies depending on the selected model of knowledge used to predict the stream quality. For the examined problem, the best model is the Regression model. It allows us to achieve a minimum reduction of the quality of a single stream. This is explaining to the properties of the usable method. It allows you to build a model of knowledge, which is flexible.

\section{Conclusions}

The proposed approach in article of dynamic control flow measurements can significantly reduce network traffic and distribute the computational load on the entire collection of measurement environment. For it is proposed to use the technology of cloud computing and machine learning techniques. Transfer of quality assessment measurements on MP allows a part of calculations transfer from the measurement processing center to the MP. Using machine learning methods for quality prediction allows determining the need for transmission measurements and thereby avoiding data transfer, not included in a single stream.

As a result, it is possible to reduce the amount of transmitted information in several times. This allows using for transmission measurements channels with low bandwidth. Besides it is reduced delivery time measurements to decision makers. This is particularly important for facilities controlled and managed from processing center.

\section{Acknowledgements}

This work was supported by the Ministry of Education and Science of the Russian Federation in the framework of the state order "Organization of Scientific Research", Task \#2.6113.2017/6.7.

\section{References}

[1] Tsai C.-W., Lai C.-F., Vasilakos A. V. Future internet of things: open issues and challenges. Wireless Networks, Vol. 20, Issue 8, 2014, p. 2201-2217.

[2] Gubbi Jayavardhana, Buyya Rajkumar, Marusic Slaven, Palaniswamia Marimuthu Internet of Things (IoT): a Vision, Architectural Elements, and Future Directions. Future Generation Computer Systems, Vol. 29, Issue 7, 2013, p. 1645-1660.

[3] Peter Mell, Timothy Grance The NIST Definition of Cloud Computing. Recommendations of the National Institute of Standards and Technology. Computer Security Division Information Technology Laboratory National Institute of Standards and Technology, Gaithersburg, 2011.

[4] Bonomi F., Milito R., Zhu J., Addepalli S. Fog computing and its role in the internet of things. Proceedings of MCC, Helsinki, Finland, 2012, p. 13-16.

[5] Barsegian Armenak, Kupriyanov Mikhail, Kholod Ivan, Yelizarov Sergey, Thess Michael Analysis of Data and Processes: From Standard to Realtime Data Mining. Re Di Roma-Verlag, 2014, p. 299.

[6] Witten I. H., Eibe F., Hall Mark A. Data Mining: Practical Machine Learning Tools and Techniques. 3nd Edition. Morgan Kaufmann Publishers, San Francisco, 2011, p. 629.

[7] Rucker Derek D., McShane Blakeley B., Preacher Kristopher J. A Researcher's Guide to Regression, Discretization, and Median Splits of Continuous Variables. Northwestern University, USA, 2015.

[8] Quinlan J. R. Learning with Continuous Classes. University of Sydney, Australia, 2006. 